\title{
重大林业入侵害虫美国白蛾对植物次生挥发物质的 触角电位活性
}

\author{
唐点 $^{(12)}$ ，苏茂文 ${ }^{(12)}$ ，张钟宁 ${ }^{(1 *}$ \\ (1) 中国科学院动物研究所, 农业虫鼠害综合治理研究国家重点实验室, 北京 100101; \\ (2) 中国科学院研究生院, 北京 100049 \\ * 联系人, E-mail: zhangzn@ioz.ac.cn \\ 2012-03-01 收稿, 2012-06-11 接受 \\ 国家重点基础研究发展计划(2009CB119204)资助
}

\begin{abstract}
摘要 在室内分别对美国白蛾䧳虫和雄虫进行了55 种寄主化合物的触角电位活性实验. 结果 显示, 雄蛾反应较强的化合物分别是(强度从大到小排列，下同): 反-2-已烯乙酸酯、壬醛、已醛、 反-2-已烯醛、已醇、乙酸异戊酯、顺-3-已烯乙酸酯、乙酰乙酸乙酯和香茅醛及(+)-香茅醛; 雌 蛾反应较强的化合物分别是: 已醇、已醛、苯乙酮、乙酸异戊酯、壬醛、反-2-已烯醛、顺-3-已 烯乙酸酯、香茅醛、顺-3-已烯-1-醇及反-2-已烯醇. 从 55 种化合物中䇥选出了 7 种活性较高的 化合物进行了剂量反应实验, 结果显示, 美国白蛾雄蛾对 7 种化合物的反应都随着剂量的增加 而增强, 而且雄蛾对每种化合物最高剂量 $1000 \mu \mathrm{g}$ 的相对反应值都与同种化合物其他剂量的相 对反应值反应有显著性差异.
\end{abstract}

关键词

美国白蛾

触角电位

植物次生

挥发物质

入侵害虫
寄主植物对食叶昆虫的交配和繁殖活动有着重 要的影响, 这种影响表现在昆虫的生理和行为两方 面, 即性信息素信号交流 ${ }^{[1]}$. 如有的昆虫从寄主植物 获得某种化合物并将其作为性信息素化合物或化合 物的前体物质 ${ }^{[2]}$; 有的昆虫只有在感受到寄主植物的 气味时, 才产生或释放性信息素 ${ }^{[3]}$. 寄主植物对食叶 昆虫两性行为的影响反映出了昆虫为了尽可能实现 两性间的求偶和繁殖所采取的策略.

寄主植物化合物通常能够增强昆虫对性信息素 的反应, 目前已有不少关于植物挥发性次生物质对 昆虫性信息素具增效作用的报道. (Z)-3-己烯乙酸酯 是常见的绿叶气味之一, 对美洲棉铃虫 (Helicoverpa $z e a)$ 性信息素 ${ }^{[4]}$ 和烟芽夜蛾 (Heliothis virescens)性信 息素 ${ }^{[5]}$ 都具有增效作用. 绿叶气味混合物与苹果蛽蛾 (Cydia pomonella)性信息素混用要比单独应用性信息 素诱到更多的苹果冨蛾雄蛾 ${ }^{[6]}$.

美国白蛾是世界性检疫害虫, 属鳞翅目, 灯蛾科.
自 1979 年人侵中国以来, 造成了广泛危害, 其寄主 非常广泛, 可危害上百种植物, 但是其亦有取食偏好, 最喜食植物为桑树、糖槭、杨树、臭椿等 ${ }^{[7]}$. 美国白 蛾的性信息素鉴定工作早在 20 世纪 70 年代初就在进 行, 目前已经公认的美国白蛾性信息素成分具有 4 个 主要组分 ${ }^{[8]}$, 我国的美国白蛾性信息素鉴定工作由 $\mathrm{Su}$ 等人 ${ }^{[7]}$ 于 2008 年完成. 植物挥发物质与昆虫间的 交互作用研究是化学生态学的一个重要课题 ${ }^{[9]}$, 然而 美国白蛾与寄住植物之间通过化合物进行的交互作 用却鲜有报道 ${ }^{[10]}$. 我们认为, 不同的植物源挥发次 生气味物质成分, 对美国白蛾定位寄主植物产生了 影响, 从而产生了寄主偏好. 基于对鳞翅目昆虫的取 食和趋向活性化合物 ${ }^{[11]}$ 以及美国白蛾宿主的挥发性 化合物研究 ${ }^{[12]}$ 的相关文献和资料检索, 我们选择了 55 种对鳞翅目具有引诱作用的化合物, 在室内对美 国白蛾雌雄成虫进行了触角电位的生理活性实验, 旨在篮选可用于监控美国白蛾信息素的增效剂并探

英文版见: Tang R, Su M W, Zhang Z N. Electroantennogram responses of an invasive species fall webworm (Hyphantria cunea) to host volatile compounds Chin Sci Bull, 2012, 57, doi: 10.1007/s11434-012-5356-z 
讨寄主植物挥发物对美国白蛾在我国成功人侵中的 可能作用.

\section{1 材料与方法}

（i ）实验昆虫. 于 2009 年 5 月在河北省三河市 桑树 $(M . a l b a)$ 上采集美国白蛾幼虫, 在实验室内以桑 树叶饲养至化蛹, 光照周期为 $16 \mathrm{~h}$ 光照: $8 \mathrm{~h}$ 光照, 温 度为 $25 \pm 1^{\circ} \mathrm{C}$. 根据蛹体腹部的形态特征将雌雄蛹分开, 置于同幼虫饲养相同的环境待其羽化. 新羽化的雌 蛾和雄蛾分开放在养虫笼 $(30 \mathrm{~cm} \times 30 \mathrm{~cm} \times 30 \mathrm{~cm})$, 于室
温下饲养, $1 \sim 3 \mathrm{~d}$ 的成虫触角用于触角电生理反应测试.

(ii) 触角电位反应测试化合物. 测试化合物的 选择是以美国白蛾寄主植物次生物质的分析和有关 对蛾类昆虫具有引诱活性的文献资料为依据. 化合 物的来源及纯度见表 1 .

(iii) 触角电位测试方法. 实验所用触角电位 (eletctroantennogram, EAG)技术, Visser ${ }^{[13]}$ 已有详尽 描述. 取羽化后 2 3 $\mathrm{d}$ 未交配成虫, 将其触角从基部 剪下, 去掉触角顶端. 在双目实体显微镜(Olympus 273059)下, 用冷光源(Intralux 5000-1)照明, 将记录

表 1 实验中所用试剂的纯度和来源

\begin{tabular}{|c|c|c|c|c|c|}
\hline 试剂名称 & 纯度(\%) & 来源 a) & 试剂名称 & 纯度(\%) & 来源 ${ }^{\text {a) }}$ \\
\hline 醇类 & & & $(-)-\beta$-蒎烯 & $\geqslant 99$ & $\mathrm{a}$ \\
\hline 3-丁炔-1-醇 & $\geqslant 97$ & $\mathrm{~b}$ & (+)- $\alpha$-蒎烯 & $\geqslant 99$ & $\mathrm{a}$ \\
\hline 正丁醇 & $\geqslant 97$ & d & (-)- $\alpha$-蒎烯 & $\geqslant 99$ & $\mathrm{a}$ \\
\hline 1,4-戊二烯-3-醇 & $\geqslant 97$ & $\mathrm{c}$ & (土)-3-蒈烯 & $\geqslant 99$ & $\mathrm{a}$ \\
\hline 顺-3-己烯-1-醇 & $\geqslant 99$ & $\mathrm{a}$ & $\beta$-石竹烯 & $\geqslant 97$ & $\mathrm{c}$ \\
\hline 反-2-已烯-1-醇 & $\geqslant 99$ & $\mathrm{a}$ & 酯类 & & \\
\hline 2-乙基-1-己醇 & $\geqslant 99$ & $\mathrm{a}$ & 乙酰乙酸乙酯 & $\geqslant 97$ & $\mathrm{~d}$ \\
\hline 2,5-己二醇 & $\geqslant 96$ & $\mathrm{c}$ & 乙酸异戊酯 & $\geqslant 97$ & $\mathrm{~d}$ \\
\hline 正己醇 & $\geqslant 97$ & d & 顺-3-己烯乙酸酯 & $\geqslant 99$ & $\mathrm{e}$ \\
\hline 正庚醇 & $\geqslant 97$ & $\mathrm{~d}$ & 反-2-己烯乙酸酯 & $\geqslant 99$ & $\mathrm{e}$ \\
\hline 正辛醇 & $\geqslant 97$ & $\mathrm{~d}$ & 酮类 & & \\
\hline 正癸醇 & $\geqslant 97$ & d & 3-戊酮 & $\geqslant 97$ & $\mathrm{~b}$ \\
\hline 顺,顺-9,12-十八碳二烯醇 & $\geqslant 95$ & $\mathrm{~b}$ & (+)-2-茨酮 & $\geqslant 97$ & $\mathrm{~b}$ \\
\hline 橙花叔醇 & $\geqslant 95$ & $\mathrm{~b}$ & (-)-2-茨酮 & $\geqslant 97$ & $\mathrm{~b}$ \\
\hline 香叶醇 & $\geqslant 97$ & $\mathrm{~b}$ & 芳香族化合物 & & \\
\hline 醛类 & & & 苯甲醛 & $\geqslant 96$ & $\mathrm{~d}$ \\
\hline 反-2-己烯醛 & $\geqslant 99$ & $\mathrm{a}$ & 苯乙酮 & $\geqslant 95$ & $\mathrm{~d}$ \\
\hline 己醛 & $\geqslant 97$ & a & 对异丙基甲苯 & $\geqslant 97$ & $\mathrm{a}$ \\
\hline 壬醛 & $\geqslant 97$ & a & 2-苯乙醇 & $\geqslant 97$ & $\mathrm{~d}$ \\
\hline 糠醛 & $\geqslant 97$ & $\mathrm{a}$ & 枯茗醛 & $\geqslant 97$ & $\mathrm{c}$ \\
\hline 香茅醛 & $\geqslant 97$ & $\mathrm{~b}$ & 1-甲基菜 & $\geqslant 97$ & $\mathrm{c}$ \\
\hline (+)-香茅醛 & $\geqslant 97$ & $\mathrm{~b}$ & 水杨酸甲酯 & $\geqslant 97$ & $\mathrm{~d}$ \\
\hline 酸类 & & & 水杨酸乙酯 & $\geqslant 97$ & $\mathrm{~d}$ \\
\hline 丙酸 & $\geqslant 95$ & $\mathrm{~d}$ & 胺(氨)类 & & \\
\hline 烯(萜)类 & & & 尸胺 & $\geqslant 99$ & $\mathrm{e}$ \\
\hline 2,6-二甲基-2,4,6-辛三烯 & $\geqslant 99$ & $\mathrm{~b}$ & 腐胺 & $\geqslant 99$ & $\mathrm{e}$ \\
\hline$\alpha$-萜品烯 & $\geqslant 97$ & c & 二乙胺 & $\geqslant 97$ & $\mathrm{~d}$ \\
\hline 异松油烯 & $\geqslant 97$ & $\mathrm{c}$ & 乙二胺 & $\geqslant 97$ & $\mathrm{~d}$ \\
\hline (+)-长叶烯 & $\geqslant 99$ & a & 甲胺 & $\geqslant 33$ & $\mathrm{~d}$ \\
\hline 月桂烯 & $\geqslant 97$ & $\mathrm{~b}$ & 氨水 & $\geqslant 25$ & $\mathrm{~d}$ \\
\hline (-)-柠檬烯 & $\geqslant 97$ & a & 其他 & & \\
\hline (-)-葫烯 & $\geqslant 98$ & $\mathrm{a}$ & 石蜡油 & 化学纯 & $\mathrm{f}$ \\
\hline (土)-茨烯 & $\geqslant 98$ & $\mathrm{a}$ & & & \\
\hline
\end{tabular}

a) a, Aldrich; b, Fluka; c, Sigma; d, 北京医药化学制品有限责任公司; e, 日本东京化成工业株式会社; f, 天津市福晨化学试剂厂 
电极套在触角的顶端, 参考电极套在触角基部上. 玻 璃微电极内灌注生理盐水, 银-氯化银丝插人玻璃微 电极内并连接到微动操作仪(Syn-tech MP-15)上的输 人探针, 后者连接到交流/直流放大器 (Syntech UN06), 其信号输出端与计算机相连. 其中所用的数据 处理软件 Spike 由 Syntech 公司提供.

刺激气体控制装置(Syntech CS-05)将气体用活 性碳净化、压缩产生持续气流，通过气味混合管吹过 触角, 气体流量为 $30 \mathrm{~mL} \mathrm{~s}^{-1}$, 气味混合管口正对触 角, 相距约 $1 \mathrm{~cm}$. 取刺激样品 $10 \mu \mathrm{L}$ 滴于巴斯德管内 的滤纸 $(0.5 \mathrm{~cm} \times 5 \mathrm{~cm})$ 上, 管口一端连接刺激气体控 制装置, 另一端插人气味混合管上的侧孔, 用脚踏板 人为控制刺激, 每次刺激时间为 $0.1 \mathrm{~s}$, 两次刺激间隔 时间为 $1 \mathrm{~min}$, 以使触角状态恢复. 所有化合物皆用 石蜡油(paraffin oil)稀释, 浓度为 $100 \mathrm{mg} \mathrm{mL}$, 用于 测试剂量反应的化合物浓度梯度为 $0.1,1,10$ 和 100 $\mathrm{mg} \mathrm{mL} \mathrm{m}^{-1}$. 每次刺激剂量为 $10 \mu \mathrm{L} \times(0.1,1,10,100$ $\mathrm{mg} \mathrm{mL}^{-1}$ ), 分别为 $1,10,100,1000 \mu \mathrm{g}$. 以 $10 \mu \mathrm{L}$ 的石 蜡油为空白对照.

选用壬醛为标准化合物，测量时刺激顺序为空 白对照、壬醛、样品(低浓度到高浓度)、空白对照、壬 醛, 每根触角重复 3 次. 每种化合物重复 10 根来自不 同昆虫个体的触角. 样品的触角电位反应值按照如 下公式进行校正，以消除溶剂和环境因素的干扰：

样品校正触角电位反应值 $=R_{\mathrm{c}}-\left(R_{\mathrm{C}-1}-R_{\mathrm{C}+1}\right) / 2$, 其中, $R_{\mathrm{C}}$ 为所测样品触角电位的反应值; $R_{\mathrm{C}-1}$ 为测试
样品前空白对照的触角电位反应值; $R_{\mathrm{C}+1}$ 为测试样品 后空白对照的触角电位反应值. 取各样品校正触角 电位反应值与标准化合物校正触角电位反应值作百 分比进行比较, 用 Duncun's 复极式方差检验(ANOVA, SPSS 13.0 for Windows)来分析各处理平均值间的差 异显著性 $(P<0.05)$; 雌蛾和雄蛾对同一种化合物的相 对反应值用 $t$-检验(SPSS 13.0 for Windows)分析其差 异显著性。

\section{2 结果}

\section{1 美国白蛾成虫对醇类化合物的触角电生理反应}

图 1 所示为美国白蛾对醇类化合物的触角电位 反应结果, 在所测试的醇类化合物中, 美国白蛾雌蛾 对正己醇的 EAG 反应最强, 其反应值超过了对照化 合物壬醛，与壬醛没有显著性差异但与其他醇类化 合物都存在显著性的差异 $(P<0.05)$; 其次分别是正庚 醇、顺-3-己烯-1-醇、反-2-已烯-1-醇和 2-乙基-1-已醇, 这四者之间没有显著性的差异; 正丁醇、正辛醇、香 叶醇的反应处于中间水平，三者之间没有显著性差 异; 其余的醇类化合物反应值较低, 不及壬醛的四分 之一; 美国白蛾雌蛾对 1,4-戊二烯-3-醇的 EAG 反应 为负值 $(N=30)$.

美国白蛾雄蛾对正己醇的 $\mathrm{EAG}$ 反应也是最强, 其反应值(相对比 79.34\%)不如对照化合物壬醛, 并 存在显著性的差异，同时与其他醇类化合物相比亦

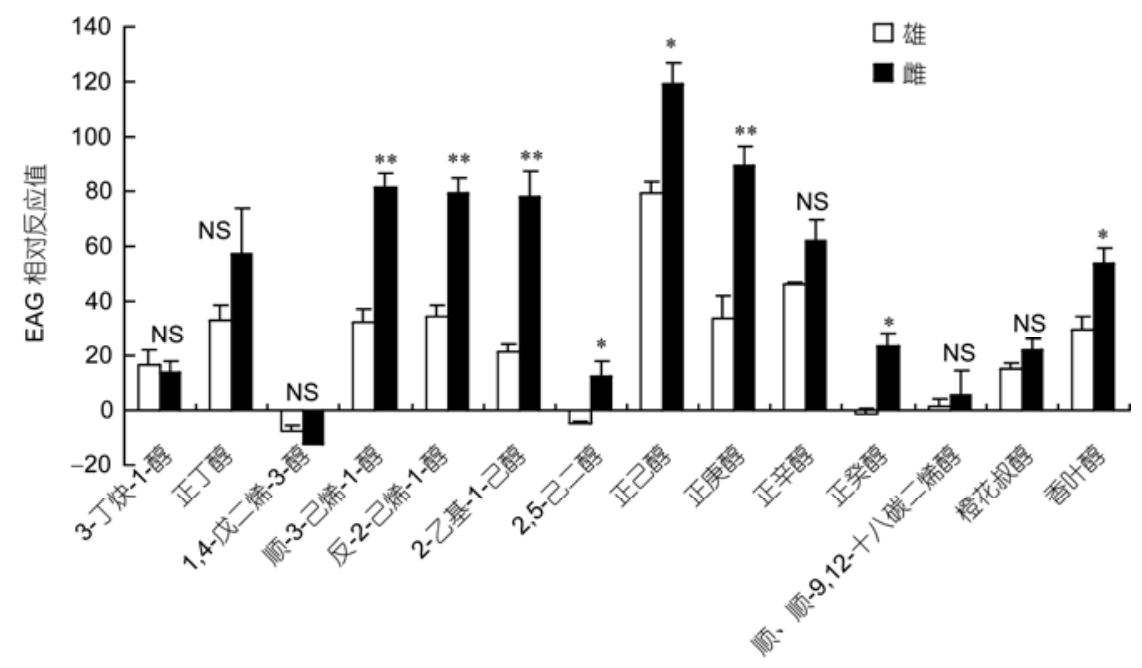

图 1 美国白蛾雌蛾和雄蛾对醇类化合物的触角电生理反应比较 $\mathrm{NS}$, 差异不显著; *, 差异显著, $P<0.05$; **, 差异极显著, $P<0.01$, $t$-检验(SPSS 13.0). 下同 
皆存在明显的差异 $(P<0.05)$; 其次是正辛醇, 其反应 值与其他醇类化合物相比亦都存在明显的差异 $(P<0.05)$; 其余的醇类化合物反应值较低, 都不及壬 醛的 35\%; 而且美国白蛾雄蛾对 1,4-戊二烯-3-醇、2,5己二醇、正癸醇的 $\mathrm{EAG}$ 反应为负值 $(N=30)$.

\section{2 美国白蛾成虫对醛类和酸类化合物的触角电 生理反应}

图 2 所示为美国白蛾对醛类和酸类化合物的触 角电位反应结果. 就所试的醛类和酸类化合物而言, 美国白蛾䧳蛾对已醛的 EAG 反应最强, 其次是壬醛 和反-2-己烯醛, 三者之间没有显著性差异; 但是三 者与其他醛类化合物存在显著性的差异 $(P<0.05)$; 糠 醛、香茅醛和(+)-香茅醛三者之间没有显著性的差异, 雌蛾对三者的 EAG 相对反应值都在 $64 \%$ 以上; 雌蛾 对丙酸的反应值不高, 但是由于酸类化合物只测试 了丙酸一种化合物, 因此不能断言雌蛾对酸类化合 物不敏感, 美国白蛾雌蛾对所试醛类的反应显著地 强于对丙酸的反应.

美国白蛾雄蛾对壬醛的 EAG 反应最强, 其次是 正己醛和反-2-己烯醛，三者之间没有显著性差异; 但是三者与其他醛类化合物存在显著性的差异 $(P<0.05)$; 糠醛、香茅醛和 $(+)$ - 香茅醛三者之间没有显 著性的差异, 但雄蛾对三者的 EAG 相对反应值都在 45\%以下; 与雌蛾类似, 雄蛾对丙酸的反应值不高, 除了糠醛以外, 雄蛾对所试醛类的反应皆强于对丙 酸的反应. 我们发现, 除了糠醛, 美国白蛾雌蛾和雄 蛾对其他醛类的反应之间没有显著性的差异.

美国白蛾雌蛾对所试的醛类化合物的反应普遍 要比雄蛾敏感, 反应值相对较高; 美国白蛾雌蛾和雄 蛾都对正己醛、壬醛和反-2-已烯醛的反应较为强烈,
相对比都在 $93 \%$ 以上, 而对丙酸的反应值都比较弱, 显示出了对丙酸的不敏感性.

\section{3 美国白蛾成虫对萜烯类化合物的触角电生理 反应}

图 3 所示为美国白蛾对萜烯类化合物的触角电 位反应结果. 就所试的萜烯类化合物而言, 美国白蛾 雌蛾对 2,6-二甲基-2,4,6-辛三烯的 EAG 反应最强, 其 次是异松油烯, 二者之间没有显著性差异, 但是二者 与壬醛之间存在明显的差异 $(P<0.05)$; 雌蛾对其他萜 烯类化合物 $\mathrm{EAG}$ 相对反应值都在 $46 \%$ 以下, 尤其 $(+)-$ 长叶烯和 $(-)-\alpha$-蒎烯出现了负值 $(N=30)$.

美国白蛾雄蛾对所试的萜烯类化合物普遍反应 都低, 反应值相对最高的化合物为 $\alpha$-萜品烯, 其 EAG 相对反应值仅有 $23.33 \%$. 壬醛与所有测试的萜 烯类化合物之间都存在明显的差异 $(P<0.05)$; 同雌蛾 一样, 雄蛾对 $(-)-\alpha$-蒎烯的反应亦出现了负值 $(N=30)$.

我们发现, 美国白蛾䧳蛾和雄蛾对 2,6-二甲基2,4,6-辛三烯、 $\alpha$-萜品烯、异松油烯和 $\beta$-石竹烯的反 应存在显著性的差异.

美国白蛾雌蛾和雄蛾对所试的萜烯类化合物反 应相对都比较弱, 美国白蛾雌蛾的反应普遍要比雄 蛾敏感, 反应值相对较高; 美国白蛾雌蛾和雄蛾都对 $(-)-\alpha$-蒎烯出现了负反应, 而对不同构型的 $(+)-\alpha$-蒎 烯和(-)- $\beta$-蒎烯反应并不是负值, 表明了美国白蛾对 化合物的不同异构体具有不同的选择性.

\section{4 美国白蛾成虫对酯类和酮类化合物的触角电 生理反应}

图 4 所示为美国白蛾对酯类和酮类化合物的触 角电位反应结果, 美国白蛾雌蛾对所试的酯类化合

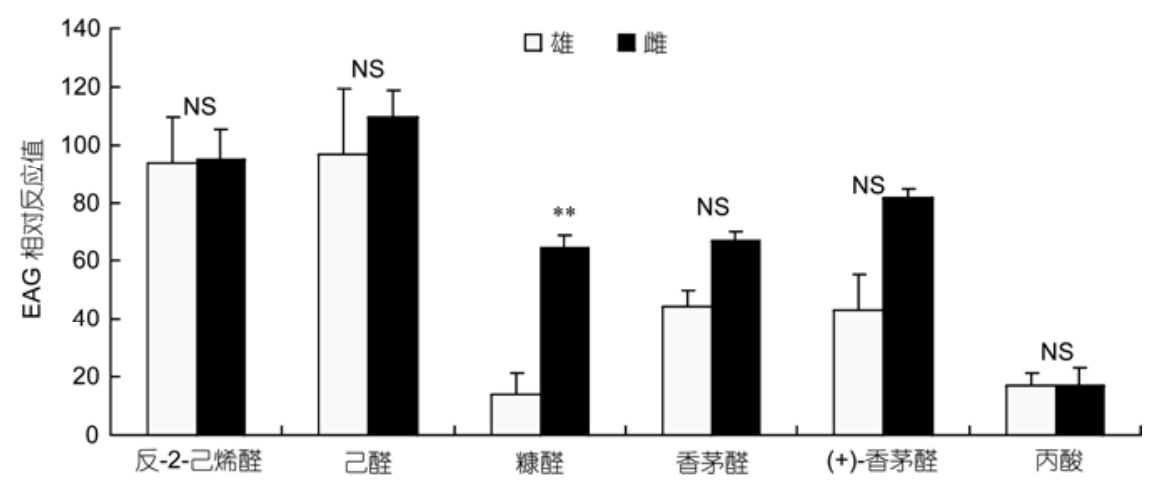

图 2 美国白蛾雌蛾和雄蛾对醛类和酸类化合物的触角电生理反应比较 


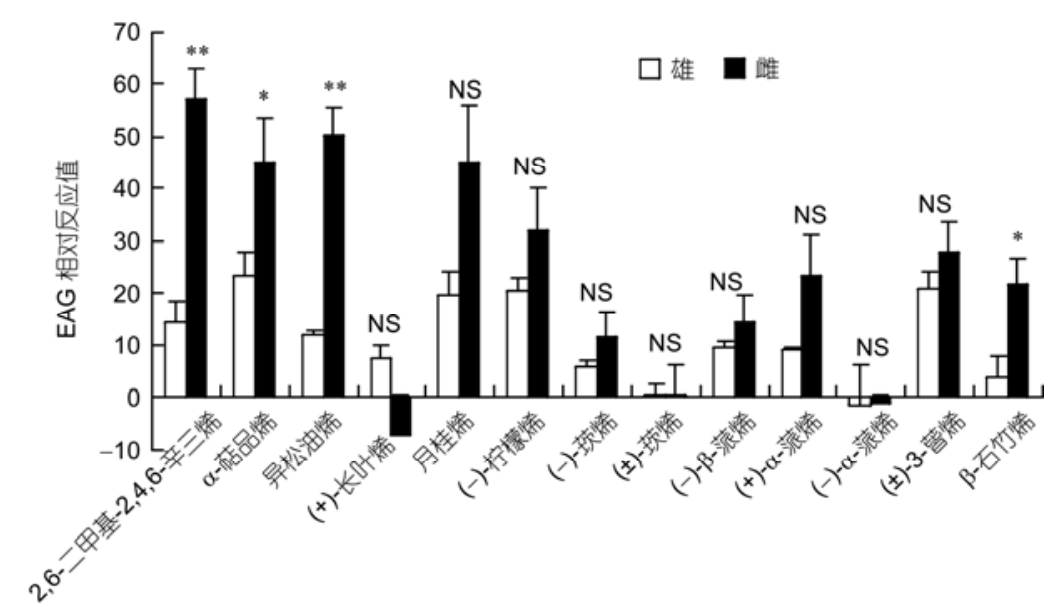

图 3 美国白蛾䧳蛾和雄蛾对萜烯类化合物的触角电生理反应比较

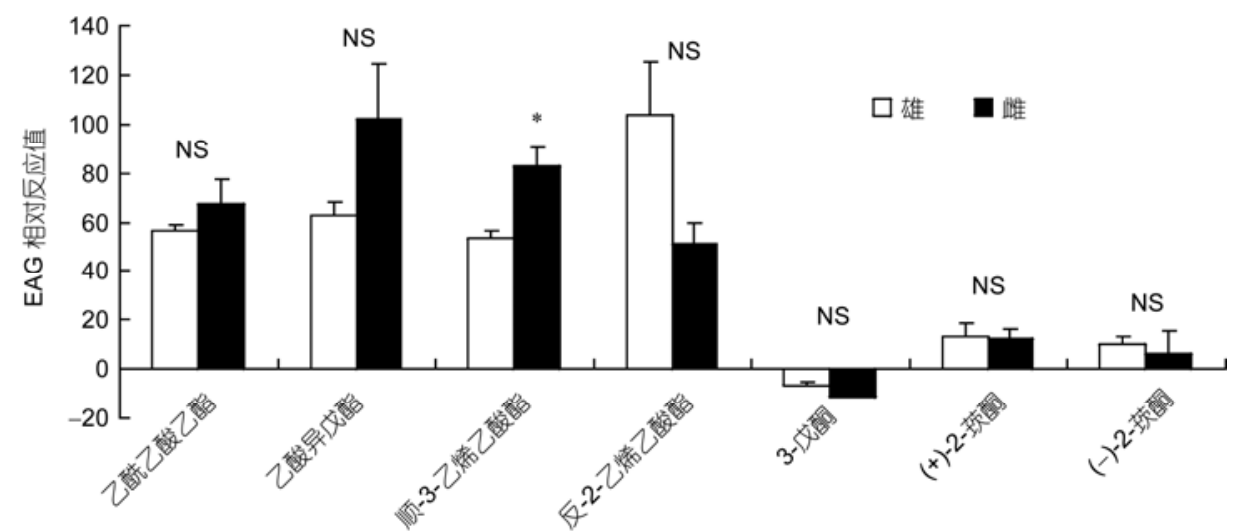

图 4 美国白蛾雌蛾和雄蛾对酯类和酮类化合物的触角电生理反应比较

物要比酮类化合物敏感. 对乙酸异戊酯的 EAG 反应 最强, 其反应值超过了壬醛，与壬醛没有显著性差异; 其次分别是顺-3-己烯乙酸酯、乙酰乙酸乙酯和反-2己烯乙酸酯, 三者之间没有显著性的差异, 它们的 EAG 相对反应值都超过了 51\%; 美国白蛾雌蛾对酮 类化合物的 EAG 反应较弱, 其相对反应值都低于 $13 \%$, 对 3-戊酮的反应为负值 $(N=30)$.

美国白蛾雄蛾对所试的酯类化合物比酮类化合 物敏感. 对反-2-已烯乙酸酯的 EAG 反应最强, 其反 应值超过了壬醛, 与壬醛没有显著性差异, 但与其他 所测试的酯类都存在显著性的差异 $(P<0.05)$; 其次分 别是乙酸异戊酯、顺-3-己烯乙酸酯和乙酰乙酸乙酯, 三者之间没有显著性的差异, 它们的 EAG 相对反应 值都超过了 $56 \%$; 同雌蛾相似, 雄蛾对酮类化合物的 EAG 反应一样较弱, 其相对反应值都低于 $14 \%$, 对 3 -戊酮的反应亦为负值 $(N=30)$.
我们发现, 除了顺-3-已烯乙酸酯, 美国白蛾雌蛾 和雄蛾对所测试的其他酯类和酮类化合物的反应皆 不存在差异.

美国白蛾雌蛾和雄蛾对所试的酯类化合物比酮 类化合物敏感, 美国白蛾雌蛾和雄蛾的 EAG 反应最 强的化合物有所不同, 雌蛾对乙酸异戊酯最敏感, 而 雄蛾对反-2-己烯乙酸酯最敏感, 雌蛾和雄蛾对乙酸 异戊酯及反-2-己烯乙酸酯的反应没有显著性差异.

\section{5 美国白蛾成虫对芳香族化合物的触角电生理 反应}

图 5 所示为美国白蛾对芳香族化合物的触角电位 反应结果, 就所测试的芳香族化合物而言, 美国白蛾 雌蛾对苯乙酮的 EAG 反应最强, 其反应值超过了壬 醛, 与壬醛没有显著性差异, 与其他所测试的芳香族 化合物都存在显著性的差异 $(P<0.05)$; 其次分别是苯 


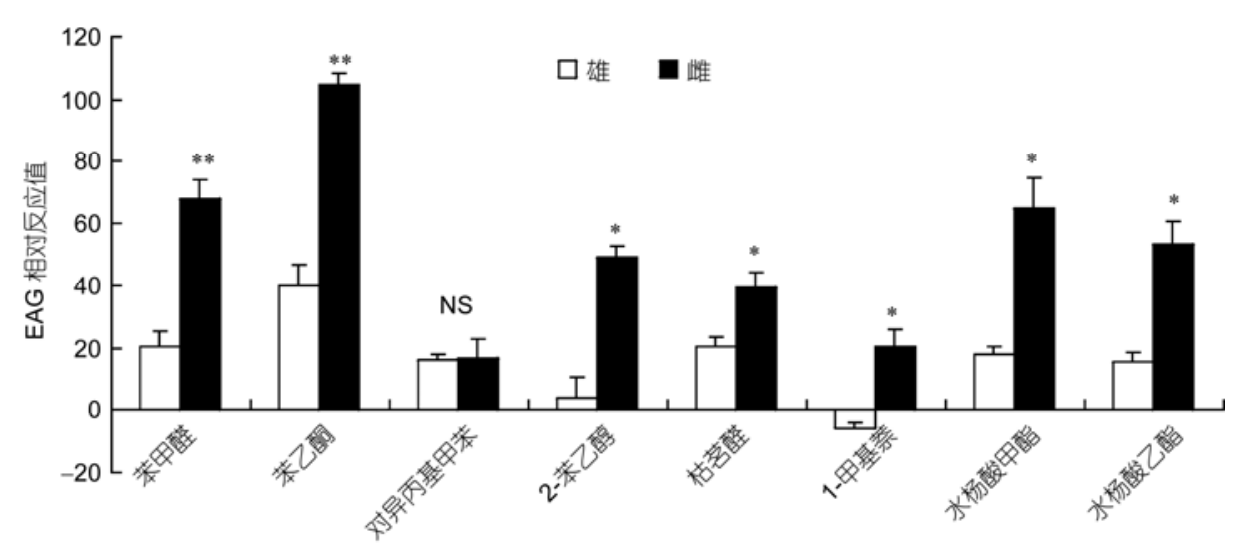

图 5 美国白蛾雌蛾和雄蛾对芳香族化合物的触角电生理反应比较

甲醛和水杨酸甲酯, 二者之间没有显著性的差异, 它 们的 EAG 相对反应值都超过了 64\%; 美国白蛾雌蛾 对对异丙基甲苯的 EAG 反应最弱, 其相对反应值低 于 $17 \%$.

美国白蛾雄蛾对苯乙酮的 EAG 反应也是最强, 但其相对反应值仅为 $40 \%$, 与壬醛存在显著性差异 $(P<0.05)$, 与其他所测试的芳香化合物都存在显著性 的差异 $(P<0.05)$; 其次分别是苯甲醛、枯芳醛、水杨 酸甲酯、对异丙基甲苯和水杨酸乙酯, 五者之间没有 显著性的差异, 但是它们的 EAG 相对反应值都低于 21\%; 美国白蛾雄蛾对 1-甲基萗的 EAG 反应为负值 $(N=30)$.

除了对异丙基甲苯, 美国白蛾雌蛾和雄蛾对所 测试的其他芳香族化合物的反应皆有显著性的差异.

美国白蛾雌蛾对所试的芳香族化合物要比雄蛾 相对敏感, 反应值相对较高; 美国白蛾雌蛾和雄蛾都 对苯乙酮的反应最为强烈, 而对对异丙基甲苯和 1甲基荎反应较弱, 尤其雄蛾对 1-甲基荎出现了负值.

\section{6 美国白蛾成虫对胺(氨)类化合物的触角电生 理反应}

图 6 所示为美国白蛾对胺(氨)类化合物的触角电 位反应结果, 就所测试的胺(氨)类化合物而言, 美国 白蛾雌蛾对腐胺的 $\mathrm{EAG}$ 反应最强, 其次是尸胺、二 乙胺和甲胺, 四者之间没有显著性差异, 但四者与壬 醛存在显著性差异 $(P<0.05)$; 美国白蛾䧳蛾对乙二胺 和氨水的 $\mathrm{EAG}$ 反应较弱, 尤其对乙二胺是负值 $(N=$ 30).

美国白蛾雄蛾亦是对腐胺的 $\mathrm{EAG}$ 反应最强, 与 壬醛存在显著性差异 $(P<0.05)$; 美国白蛾雌蛾对其他 胺(氨)类化合物的反应都很弱, 尤其对乙二胺、甲胺 和氨水的 $\mathrm{EAG}$ 反应为负值 $(N=30)$.

除了乙二胺和氨水, 美国白蛾雌蛾和雄蛾对所 测试的其他胺类化合物的反应皆有显著性的差异.

美国白蛾雌蛾和雄蛾对所试的胺(氨)类化合物 相对都比较弱, 雌蛾的反应要比雄蛾相对敏感, 反应

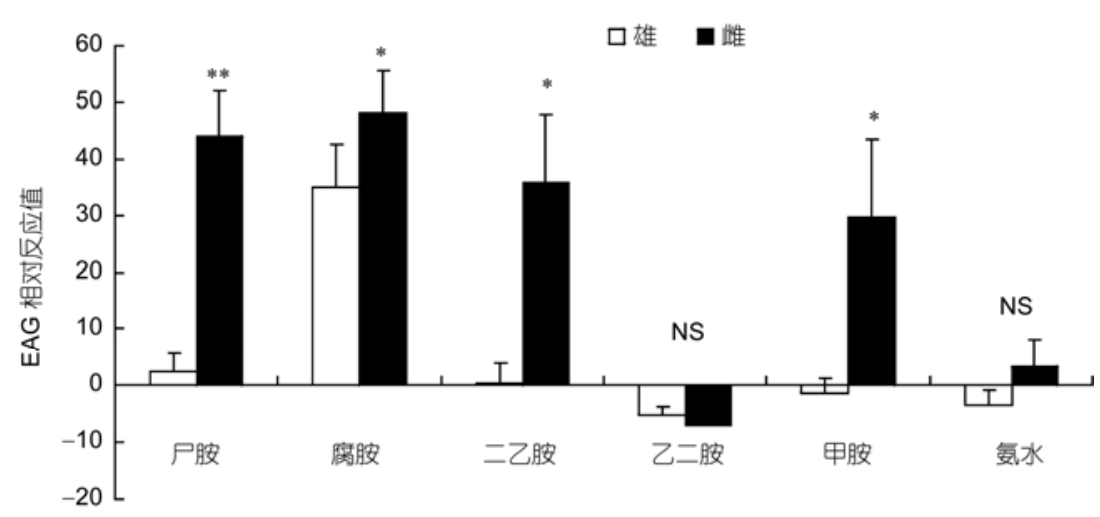

图 6 美国白蛾雌蛾和雄蛾对胺(氨)类化合物的触角电生理反应比较 
值相对较高; 美国白蛾雌蛾和雄蛾都对腐胺的反应 最为强烈, 同时对乙二胺皆出现了负值, 尤其雄蛾对 甲胺和氨水亦出现了负值.

\section{7 美国白蛾雄蛾对几种化合物的剂量反应}

通过以上研究我们篮选出了一些对美国白蛾雄 蛾具有较强 EAG 活性的化合物, 按照反应强度高低 的顺序分别是反-2-已烯乙酸酯>壬醛>己醛>反-2-己 烯醛>己醇>乙酸异戊酯>顺-3-己烯乙酸酯, 同时我们 选择了这 7 种高活性的化合物以研究美国白蛾雄蛾对 它们的剂量反应.

美国白蛾雄蛾对 7 种化合物的反应都是随着剂 量的增加而增强, 而且雄蛾对每种化合物最高剂量 $1000 \mu \mathrm{g}$ 的相对反应值都与同种化合物其他剂量的相 对反应值反应有显著性的差异; 反-2-已烯乙酸酯、正 己醛和反-2-己烯醛三者在剂量为 $1,10,100 \mu \mathrm{g}$ 的情 况下，雄蛾对这三种剂量的反应没有显著性的差异; 壬醛的 4 种剂量之间都相互存在显著性的差异; 已 醇、乙酸异戊酯和顺-3-己烯乙酸酯在 1 和 $10 \mu \mathrm{g}$ 剂量 下没有显著性的差异, 但这两种剂量与 $100 \mu \mathrm{g}$ 剂量 皆存在显著性的差异(乙酸异戊酯除外, 其在剂量 10 和 $100 \mu \mathrm{g}$ 之间差异不显著) (表 2).

\section{3 结论}

本研究测定了 55 种化合物对美国白蛾雌蛾和雄 蛾的 EAG 反应, 发现雄蛾反应较强的化合物分别是 (按照反应强度大小排列): 反-2-已烯乙酸酯、壬醛、己 醛、反-2-己烯醛、己醇、乙酸异戊酯、顺-3-己烯乙 酸酯、乙酰乙酸乙酯、香茅醛, (+)-香茅醛; 雌蛾反应 较强的化合物分别是 (按照反应强度大小排列): 已 醇、已醛、苯乙酮、乙酸异戊酯、壬醛、反-2-己烯 醛、顺-3-已烯乙酸酯、香茅醛、顺-3-己烯-1-醇、反2-已烯醇.

对美国白蛾成虫具有较强 EAG 活性的化合物多 是些脂肪族的衍生物, 尤其是以 6 个碳的醛、醇和酯 为主的绿叶气味. 通常, 无论化合物末端是何官能团, 大多数的昆虫对 6 个碳的直链化合物要比 5,7 和 8 个碳的直链化合物有更强的触角电生理反应 ${ }^{[13 \sim 16]}$. 比如, 脂肪醇对棉铃虫 (Heliothis armigera) 处女雌蛾 激发明显的 $\mathrm{EAG}$ 反应, 反应的强度因化合物碳链长 度有所不同, 以 1-已醇的反应强度最大. Burguiere 等 人 ${ }^{[17]}$ 认为棉铃虫雌蛾对 1 -已醇的反应如此强烈的原
表 2 美国白蛾雄蛾对不同剂量的 7 种化合物的触角电

生理反应

\begin{tabular}{|c|c|c|}
\hline 化合物 & 剂量 $(\mu \mathrm{g})$ & 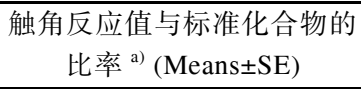 \\
\hline \multirow{4}{*}{ 反-2-己烯乙酸酯 } & 1 & $4.06 \pm 1.31 \mathrm{~b}$ \\
\hline & 10 & $24.29 \pm 4.91 \mathrm{~b}$ \\
\hline & 100 & $28.82 \pm 2.98 \mathrm{~b}$ \\
\hline & 1000 & $103.84 \pm 21.64 \mathrm{a}$ \\
\hline \multirow{4}{*}{ 壬醛 } & 1 & $0.16 \pm 0.38 \mathrm{~d}$ \\
\hline & 10 & $4.72 \pm 1.28 \mathrm{c}$ \\
\hline & 100 & $24.09 \pm 1.35 \mathrm{~b}$ \\
\hline & 1000 & $100 \mathrm{a}$ \\
\hline \multirow{4}{*}{ 正己醛 } & 1 & $2.02 \pm 0.05 b$ \\
\hline & 10 & $10.42 \pm 0.25 b$ \\
\hline & 100 & $19.12 \pm 1.01 \mathrm{~b}$ \\
\hline & 1000 & $96.52 \pm 22.77 \mathrm{a}$ \\
\hline \multirow{4}{*}{ 反-2-己烯醛 } & 1 & $7.17 \pm 2.39 b$ \\
\hline & 10 & $18.93 \pm 5.25 \mathrm{~b}$ \\
\hline & 100 & $27.00 \pm 1.78 \mathrm{~b}$ \\
\hline & 1000 & $93.62 \pm 15.89 \mathrm{a}$ \\
\hline \multirow{4}{*}{ 正己醇 } & 1 & $3.95 \pm 1.20 \mathrm{c}$ \\
\hline & 10 & $9.42 \pm 0.54 \mathrm{c}$ \\
\hline & 100 & $27.88 \pm 0.46 \mathrm{~b}$ \\
\hline & 1000 & $79.34 \pm 4.37 \mathrm{a}$ \\
\hline \multirow{4}{*}{ 乙酸异戊酯 } & 1 & $6.53 \pm 1.64 c$ \\
\hline & 10 & $9.81 \pm 2.08 \mathrm{bc}$ \\
\hline & 100 & $20.57 \pm 5.54 \mathrm{~b}$ \\
\hline & 1000 & $62.44 \pm 5.78 \mathrm{a}$ \\
\hline \multirow{4}{*}{ 顺-3-已烯乙酸酯 } & 1 & $5.67 \pm 2.70 \mathrm{c}$ \\
\hline & 10 & $9.39 \pm 3.82 \mathrm{c}$ \\
\hline & 100 & $24.26 \pm 7.05 b$ \\
\hline & 1000 & $53.54 \pm 2.90 \mathrm{a}$ \\
\hline
\end{tabular}

a) 标准化合物为 $1000 \mu \mathrm{g}$ 的壬醛; 每种化合物 4 种不同的剂 量之间进行方差分析, 不同字母表示用 Duncan's 检验在 $P=0.05$ 水平上存在显著性差异

因不仅仅在于 1-已醇自身的挥发性, 更在于棉铃虫 雌蛾对 1-己醇比其他脂肪醇类化合物有更强的敏感 性. 美国白蛾雌蛾和雄蛾也都对正己醇的反应强于 正丁醇、正庚醇、正辛醇和正癸醇，印证了昆虫对 6 个碳的直链化合物要比 5,7 和 8 个碳的直链化合物有 更强的 $\mathrm{EAG}$ 反应这一规律.

美国白蛾雄蛾对正已醛和反-2-已烯醛的反应要 比相应的醇, 即正己醇和反-2-已烯醇的敏感, 表现 
出不饱和化合物比相应的饱和化合物对昆虫的 EAG 反应更强的规律 ${ }^{[16,18 \sim 20]}$, 也表明了化学官能团对美 国白蛾成虫 EAG 反应有一定影响. Burguiere 等人 ${ }^{[17]}$ 的研究结果表明, 棉铃虫雌蛾对 6 个碳的直链酮和醛 的 $\mathrm{EAG}$ 反应比醇要低, 证明了化学官能团对昆虫 $\mathrm{EAG}$ 反应的影响，同时也证明了昆虫的 EAG 反应与 化合物的挥发性并不成正相关. 同样地, 棉铃虫雌蛾 对所测试的不饱和化合物比相应的饱和化合物反应 弱, 这一结果不仅在鳞翅目的昆虫中有所报道, Guerin 和 Städler ${ }^{[21]}$ 对 3 种双翅目 (Diptera)昆虫的研究 也出现过类似的结果.

与所测试的脂肪族化合物相比, 美国白蛾雄蛾 对芳香族化合物的反应较弱, 而雌蛾反应则相对较 强, 其中苯乙酮激起的 EAG 反应尤为突出. 目前, 许多的芳香族化合物已经用作某些鳞翅目昆虫的引 诱剂, 比如从大花六道木(Abelia grandiflora) ${ }^{[22]}$ 花中 释放的苯甲醛(benzaldehyde)、苯乙醛(phenylacetaldehyde)、2-苯乙醇 (2-phenylethanol)和苯甲醇 (benzyl alcohol)的混合物对粉纹夜蛾 (Trichoplusia ni) 有较强 的引诱作用. Heath 等人 ${ }^{[23]}$ 还报道粉纹夜蛾对苯乙醛 的触角电生理反应与对苯甲醛、乙酸芐酯 (benzyl acetate)和苯乙醛三者混合物的反应强度相当. 我们 推测能够激起较强 $\mathrm{EAG}$ 反应的苯乙酮, 也许能够具 有长程引诱美国白蛾雌蛾的活性.

美国白蛾雌蛾和雄蛾对所试的萜烯类化合物反 应相对都比较弱, 其中雌蛾和雄蛾对 (-)- $\alpha$-蒎烯和 $(+)-\alpha$-蒎烯的反应有显著性的差异, 表明了同种而不 同性的昆虫对化合物的不同异构体有不同的选择性. 因此, 如果昆虫仅对化合物的某一种对映异构体有 强烈的 EAG 反应，那么应用化合物的外消旋体可能 会降低昆虫的反应值. 但是, 在自然界中, 很多情况 下植物挥发物未必就是仅存在化合物的单一对映异 构体, 所以, 在实验室开展昆虫 EAG 实验, 很多时 候也不一定非得选择纯的对映异构体 ${ }^{[17]}$.

一般而言, 与空气清新的树林相比, 在具有不良 气味的地方生长的寄主树木更易于遭到美国白蛾的 危害, 这一现象启示我们是否不良气味如一些胺类 物质, 对美国白蛾成虫也具有一定的引诱活性呢? 因此, 我们测试了一些胺(氨)类化合物对美国白蛾成 虫的 EAG 反应, 结果表明, 美国白蛾雌蛾和雄蛾对 所试的胺(氨)类化合物反应相对都比较弱. 正如没有 哪一种单一化合物能够胜任作为马铃薯麦蛾
(Phthorimaea operculella $)^{[24]}$ 识别寄主植物的化学信 号一样, 美国白蛾雌蛾对不良气味的识别亦有可能 不是单一组分. 目前, 许多的食叶昆虫都是以寄主植 物释放的特定比例的一组化合物混合物来识别寄主 植物 ${ }^{[19]}$. 例如, 5 组分的酯类化合物 (丁酸丁酯、已酸 丙酯、己酸丁酯、丁酸己酯和己酸戊酯)引诱苹果实 蝇 (Rhagoletis pomonella) ${ }^{[25]}$; 绿叶气味混合物反-2-已 烯醛、顺-3-己烯-1-醇、反-2-已烯-1-醇和顺-3-己烯乙 酸酯是马铃薯叶甲 (Leptinotarsa decemlineata) 寻找寄 主的气味信号 $[13,20,26]$. 因此美国白蛾对不良气味的识 别也可能是一组混合物.

我们的研究结果表明美国白蛾雌蛾对所测试的 化合物的 EAG 反应普遍比雄蛾强. 有关雌性昆虫对 植物挥发性次生性挥发物反应比雄性昆虫强的研究 已有过一些报道 ${ }^{[4,25,27 ~ 30]}$, 雌性昆虫因为需要产卵, 所以对于植物源气味的敏感性就要高出一些, 同时 植物源挥发物质对于雌性产卵行为也有影响 ${ }^{[31]}$. 从 功能上说, 这可能是因为鳞翅目雌性个体的触角上 含有较多数量的对植物挥发物敏感的嗅觉感受器 ${ }^{[29]}$.

绿叶气味已被认为是一种利他素 ${ }^{[32]}$. 许多的研 究表明, 雄性昆虫对性信息素的嗅觉反应极大地受 绿叶气味影响 ${ }^{[5,6,15,33]}$. 植物源次生化合物结合信息 素的定位曾报道过对棉铃虫 (Helicoverpa armigera) 具有长程的引诱增效作用 ${ }^{[34]}$. 美国白蛾雄蛾对反-2己烯乙酸酯、壬醛、己醛、反-2-己烯醛、己醇、乙 酸异戊酯和顺-3-己烯乙酸酯的 EAG 反应较强, 可能 与这些化合物能够刺激性信息素感受器有关, 而且 这些化合物可能对性信息素有着增效作用. 美国白 蛾成虫敏感的化合物中, 植物源的成分占有很大比 重，而且很多成分被认为是取食或者产卵作用诱导 的次生化合物 ${ }^{[35,36]}$, 被诱导的植物源次生物质作用 并不单一, 通常被认为与植物被动防御作用有 关 ${ }^{[35,36]}$, 但是也存在引诱某些种类昆虫的作用, 特别 是寄生蜂等植食性昆虫的天敌 ${ }^{[37]}$. 因此这些具有电 生理活性的植物源次生物质, 可以在一定程度上揭 示出美国白蛾人侵的机理，即在植物源气味物质和 信息素的协同作用下，寻找寄住与配偶，从而实现人 侵. 而具备电生理活性的一些取食诱导的化合物, 更 是暗示了美国白蛾具有更广泛的适应性，保证了其 强大的人侵能力. 另一方面, 植物源气味的化合物可 以应用来作为一种协同剂，与信息素诱芯一同使用， 从而起到增强诱捕效率的作用. 


\section{参考文献}

1 Kesselmeier J, Staudt M. Biogenic volatile organic compounds (VOC): An overview on emission, physiology and ecology. J Atmos Chem, 1999, 33: 23-88

2 Howard R W, Blomquist G J. Ecological, behavioral, and biochemical aspects of insect hydrocarbons. Annu Rev Entomol, 2005, 50: 371-393

3 Landolt P J. Host plant influences on sex pheromone behavior of phytophagous insects. Annu Rev Entomol, 1997, 42: 371-391

4 Light D M, Flath R A, Buttery R G, et al. Host-plant green-leaf volatiles synergize the synthetic sex pheromones of the corn earworm and codling moth (Lepidoptera). Chemoecology, 1993, 4: 145-152

5 Dickens J C, Visser J H, Van-Der-Pers J C N. Detection and deactivation of pheromone and plant odor components by the beet armyworm, Spodoptera exigua (Hübner) (Lepidoptera: Noctuidae). J Insect Physiol, 1993, 39: 503-516

6 Dickens J C, Smith J W, Light D M. Green leaf volatiles enhance sex attractant pheromone of the tobacco budworm, Heliothis virescens (Lep. Noctuidae). Chemoecology, 1993, 4: 175-177

7 苏茂文, 方宇凌, 陶万强, 等. 人侵害虫美国白蛾性信息素组分的鉴定和野外活性评估. 科学通报, 2008, 53: 191-196

8 Hill A S, Kovalev B G, Nikolaeva L N, et al. Sex-pheromone of the fall webworm moth, Hyphantria cunea. J Chem Ecol, 1982, 8: 383-396

9 Reddy G V, Guerrero A. Interactions of insect pheromones and plant semiochemicals. Trends Plant Sci, 2004, 9: 253-261

10 Travis H J. The effect of eastern tent caterpillar (Malacasoma americanum) infestation on fall webworm (Hyphantria cunea) selection of black cherry (Prunus serotina) as a host tree. Am Midl Nat, 2005, 153: 270-275

11 D'alessandro M, Turlings T C J. Advances and challenges in the identification of volatiles that mediate interactions among plants and arthropods. Analyst, 2006, 131: 24-32

12 Li W G, Zhang L W, Wang C, et al. Analysis of mulberry leaf volatile components by static headspace-gas chromatography-mass spectrometry (in Chinese). Canye Kexue, 2009, 35: 355-361

13 Visser J H. Electroantennogram responses of the Colorado beetle, Leptinotarsa decemlineata, to plant volatiles. Entomol Exp Appl, 1979, 25: $86-97$

14 Kozlowski M W, Visser J H. Host plant related properties of the antennal olfactory system in the oak flea weevil, Rhynchaenus quercus. electroantennogram study. Entomol Exp Appl, 1981, 30: 169-175

15 Dickens J C. Olfaction in the boll weevil Anthonomus grandis Boh. (Coleoptera Curculionidae) electroantennogram studies. J Chem Ecol, 1984, 10: 1759-1786

16 Light D M, Jang E B, Dickens J C. Electroantennogram responses of the mediterranean fruit fly, Ceratitis capitata, to a spectrum of plant volatiles. J Chem Ecol, 1988, 14: 159-180

17 Burguiere L, Marion-Poll F, Cork A. Electrophysiological responses of female Helicoverpa armigera (Hübner) (Lepidoptera; Noctuidae) to synthetic host odours. J Insect Physiol, 2001, 47: 509-514

18 Visser J H. Differential sensory perception of plant compounds by insects. Am Chem Soc Symp Ser, 1982, 208: 215-230

19 Visser J H. Host odour perception in phytophagous insects. Ann Rev Entomol, 1986, 31: 121-144

20 Thiery D, Visser J H. Masking of host plant odour in the olfactory orientation of the Colorado potato beetle. Entomol Exp Appl, 1986, 41: 165-172

21 Guerin P M, Städler E. Host odour perception in three phytophagous Diptera: A comparative study. In: Visser J H, Minks A K, eds. Proceedings of the Fifth International Symposium on Insect-Plant Relationship, Purdoc, Wageningen, 1982. 95-105

22 Haynes K F, Zhao J Z, Latif A. Identification of floral compounds from Abelia grandiflora that stimulate upwind flight in cabbage looper moths. J Chem Ecol, 1991, 17: 637-647

23 Heath R R, Landolt P J, Dueben B, et al. Identification of floral compounds of night-blooming jessamine attractive to cabbage looper moths. Environ Entomol, 1992, 21: 854-859

24 Das P D, Raina R, Prasad A R, et al. Electroantennogram responses of the potato tuber moth, Phthorimaea operculella (Lepidoptera Gelichiidae) to plant volatiles. J Biosci, 2007, 32: 339-349

25 Zhang A J, Linnjr C, Wright S, et al. Identification of a new blend of apple volatiles attractive to the apple maggot, Rhagoletis pomonella. J Chem Ecol, 1999, 25: 1221-1233

26 Visser J H, Ave D A. General green leaf volatiles in the olfactory orientation of the Colorado potato beetle, Leptinotarsa decemlineata. Entomol Exp Appl, 1978, 24: 538-549 
27 Van-Der-Pers J N C. Comparison of electroantennogram response spectra to plant volatiles in seven species of Yponomeuta and in the tortricid Adoxophyes orana. Entomol Exp Appl, 1981, 30: 181-192

28 Ramachandran R, Khan Z R, Caballero P, et al. Olfactory sensitivity of two sympatric species of rice leaf folders (Lepidoptera Pyralidae) to plant volatiles. J Chem Ecol, 1990, 16: 2647-2667

29 Raguso R A, Light D M, Pickersky E. Electroantennogram responses of Hyles lineata (Sphingidae Lepidoptera) to volatile compounds from Clarkia breweri (Onagraceae) and other moth-pollinated flowers. J Chem Ecol, 1996, 22: 1735-1767

30 Malo E A, Cruz-López L, Toledo J, et al. Behavioral and electrophysiological responses of the Mexican fruit fly (Diptera: Terphritidae) to grava volatiles. Fla Entomol, 2005, 88: 364-371

31 Hare J D. Ecological role of volatiles produced by plants in response to damage by herbivorous insects. Annu Rev Entomol, 2011, 56: $161-180$

32 Ruther J, Reinecke A, Hilker M. Plant volatiles in the sexual communication of Melolontha hippocastani response towards time-dependent bouquets and novel function of (Z)-3-hexen-1-ol as a sexual kairomone. Ecol Entomol, 2002, 27: 76-83

33 Dickens J C. Green leaf volatiles enhance aggregation pheromone of the boll weevil Anthonomus grandis. Entomol Exp Appl, 1989, 52: 191-203

34 Lu Y J, Zhang X X. Effects of interaction of plant volatiles and sex pheromone on EAG response of Helicoverpa armigera (Huebner) (in Chinese). Acta Ecol Sin, 2003, 23: 308-313

35 Kessler A, Baldwin I T. Defensive function of herbivore-induced plant volatile emissions in nature. Science, 2001, 291: 2141-2144

36 Kessler A, Baldwin I T. Plant responses to insect herbivory: The emerging molecular analysis. Annu Rev Plant Biol, 2002, 53: 299-328

37 Dicke M, Hilker M. Induced plant defences: From molecular biology to evolutionary ecology. Basic Appl Ecol, 2003, 4: 3-14 